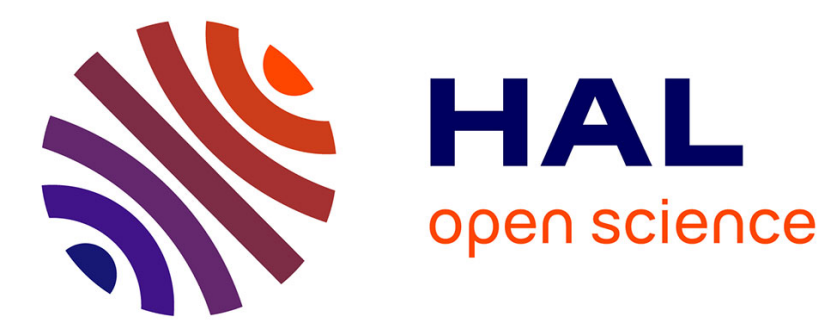

\title{
Generalized Elastic Flow Rerouting Scheme
}

Yoann Fouquet, Dritan Nace, Michal Pioro, Michael Poss, Mateusz Zotkiewicz

\section{To cite this version:}

Yoann Fouquet, Dritan Nace, Michal Pioro, Michael Poss, Mateusz Zotkiewicz. Generalized Elastic Flow Rerouting Scheme. Networks, 2015, Optimization Issues in Resilient Network Design and Modeling, 66 (4), pp.267-281. 10.1002/net.21658 • hal-01237389

\section{HAL Id: hal-01237389 \\ https://hal.science/hal-01237389}

Submitted on 30 Mar 2021

HAL is a multi-disciplinary open access archive for the deposit and dissemination of scientific research documents, whether they are published or not. The documents may come from teaching and research institutions in France or abroad, or from public or private research centers.
L'archive ouverte pluridisciplinaire HAL, est destinée au dépôt et à la diffusion de documents scientifiques de niveau recherche, publiés ou non, émanant des établissements d'enseignement et de recherche français ou étrangers, des laboratoires publics ou privés. 


\title{
Generalized Elastic Flow Rerouting Scheme
}

\author{
Yoann Fouquet*1, Dritan Nace ${ }^{\dagger 1}$, Michał Pióro ${ }^{\ddagger 2,3}$, Michael Poss ${ }^{\S 4}$, \\ and Mateusz Żotkiewicz ${ }^{\text {I2 }}$ \\ ${ }^{1}$ Sorbonne universités, Université de technologie de Compiègne, \\ CNRS, Heudiasyc UMR 7253, CS 60319, 60203 Compiègne cedex. \\ ${ }^{2}$ Institute of Telecommunications, Warsaw University of \\ Technology, Poland \\ ${ }^{3}$ Department of Electrical and Information Technology, Lund \\ University, Sweden \\ ${ }^{4}$ UMR CNRS 5506 LIRMM, Université de Montpellier 2, 161 rue \\ Ada, 34392 Montpellier Cedex 5, France
}

July 23, 2015

\begin{abstract}
The presented study deals with Elastic Flow Rerouting (EFR) - an original traffic restoration strategy for protecting traffic flows in communication networks (including wireless networks) against multiple link failures. EFR aims at alleviating the trade-off between practicability of traffic restoration and the cost of network resources observed in existing networking solutions. We present an extension of EFR capable of managing multiple partial link failures. We describe EFR and its extension, formulate the EFR related optimization problems, and discuss approaches for their resolution. We also discuss numerical results illustrating effectiveness of EFR in terms of the link capacity cost.
\end{abstract}

Keywords: survivability; traffic restoration; mixed-integer programming; partial link failures.

\section{Introduction}

This work deals with survivable communication networks, and in particular it presents a novel rerouting algorithm based on the concept of paths with

*yoann.fouquet@hds.utc.fr

†dritan.nace@hds.utc.fr

$\ddagger_{\text {michal.pioro@eit.lth.se }}$

$\S$ michael.poss@lirmm.fr

ๆ mzotkiew@tele.pw.edu.pl 
elastic, failure state-dependent flow. The purpose of the proposed algorithm is to achieve a good compromise between the restoration time management complexity on one hand and the link cost network on the other. It is also intended to be as general as possible to cope with a large number of practical cases including both total and partial link failures occurring in modern wireless networks. Both aspects are elaborated in detail below.

Let us start with some comments on the relation between management complexity of traffic restoration and traffic restoration time, and the cost of link capacity that characterizes protection/restoration strategies. In general, there is a trade-off between the amount (and thus cost) of the extra (protection) link capacity installed in the network on one hand and the traffic restoration management complexity and the restoration time required for network reconfiguration on the other hand. In other words, the more protection capacity is available the simpler and more time efficient restoration process could be applied. With this in mind, we recall below some of the well-known restoration strategies, assuming the case of total failures of single links.

In general, most of strategies designed to cope with failures of links in a network fall into one of two groups. They are either based on demand restoration or on protection. The former group, i.e., restoration strategies, is commonly divided into another two subgroups: link restoration and flow restoration. Link Restoration (LR), also called Link Protection [1], assumes single total link failures and it restores the capacity of a failed link (together with the traffic carried on the link) on a restoration path between its terminal nodes. LR achieves relatively short restoration times as compared to end-to-end flow restoration mechanisms but leads to less efficient use of link capacity.

A commonly studied flow restoration strategy is the end-to-end flow restoration utilizing stub release, called Restricted Restoration (RR) in [1,2]. The strategy restores solely the affected flows for the duration of the failure, while the unaffected flows are kept unchanged. The affected flows release the capacity that they were using on the unaffected links and this capacity can be used for rerouting - the mechanism widely known as stub release.

Another well known option for flow restoration is Global Rerouting (GR), called Unrestricted Reconfiguration in $[1,2]$. The strategy allows for the rerouting of all traffic flows, both affected and unaffected. In fact, GR, as the least constrained restoration strategy, achieves the minimum link capacity cost, but its management complexity and restoration time are the highest. With the above strategies (LR, RR, and GR), the network area involved in rerouting is gradually extended from local (vicinity of a node) to global (entire network), and at each step more efficient use of link capacity is achieved, while the restoration process itself becomes increasingly complex and its management more time consuming.

As for the methods based on protection, the most common approach is Path Diversity (PD) also known as Demand-wise Shared Protection [3]. The approach can be seen as the opposite to GR, because it does not admit any rerouting. The main assumption of PD is that the links are over-dimensioned so that there remains a sufficient amount of capacity on the unaffected paths to 
carry the traffic in any failure situation, that is to say, without any rerouting nor establishing new paths. Although PD operation is relatively simple, the network cost in terms of link capacity can increase significantly when PD is in use.

Observe that although both RR and PD admit multiple link failures, the links are assumed to fail totally (as in LR), and hence only GR is capable of dealing with partial (multiple) failures.

The second important characteristic of our method concerns its domain of applicability, covering both wired and wireless networks. In the large majority of works on network restoration for wired networks, the proposed restoration methods assume total failure of the network elements (single or multiple). Almost nothing is done to cope with partial failures despite the frequency of such events in modern wireless networks. More precisely, partial failure scenarios correspond to partial lose of transmission capacity, which happens in particular in wireless networks using microwave radio or Free Space Optical (FSO) transmission technology.

The above, somewhat simplified, considerations motivate us to look for a method with acceptable restoration time, reasonable management effort, and affordable link capacity cost. In the paper, we study a method that combines positive features of the strategies discussed above. The method is intended to achieve a compromise between the capacity cost, restoration time, and management effort, while covering a large set of applications. The method is called Elastic Flow Rerouting (EFR) and its full study, including a mathematical formulation and a discussion on its complexity, is presented in this paper. We show that the problem becomes computationally intractable even for moderate size instances, so that there is a need for heuristic approaches. Hence, we propose a heuristic solution method accompanied with numerical results. All this constitutes the first main contribution of the paper. Next, we pursue the study with an extension of the method covering multiple partial link failures scenarios. The extended EFR method combines features from both EFR and Flow Thinning [13] - another method of dealing with partial failure scenarios proposed in the past. We provide a mathematical formulation for the general case and conclude with some preliminary numerical results. This is the second main contribution of the paper.

The paper is organized as follows. In Section 2, we explain the main idea behind the EFR strategy. In Section 3, the mathematical model for the dimensioning (link cost minimization) problem is given. It is followed by a greedy heuristic method described in Section 4. We elaborate in detail how EFR is modified to handle partial failure cases and provide the corresponding mathematical model in Section 5. In Section 6, we discuss the pratical relevance of the proposed methods. In particular we focus on the application context in wireless networks and the corresponding cost model. Then, in Section 7, numerical results for both studied cases are reported. The paper is concluded in Section 8. 


\section{Elastic Flow Rerouting}

Elastic Flow Rerouting (EFR), first presented in [5], is intended to deal with total link failures. The approach can be summarized as follows. In the nominal state, when all links are operational, traffic is routed over a given set of paths for each traffic demand. In each failure state (to simplify the explanation only total failures of single links are considered), EFR uses the same set of (nominal) routing paths but with possibly modified path-flow values. In each failure state, we distinguish affected flows and affected demands. In a given failure state, the affected flows are those routed on paths going through a failing link, and the affected demands are those for which at least one path is affected and the total capacity of unaffected paths is not sufficient to carry the demand traffic. The main idea behind EFR is to restore traffic of the affected demands by means of increasing the flow on their routing paths, possibly together with decreasing flows of the unaffected demands. Thus, an increase of the pathflows is allowed only for the affected demands, while a decrease of path-flows is allowed only for the unaffected demands, besides the failed flows which are automatically decreased to 0 for both types of demands. Notice that no new paths are allowed to be established. To limit the number of path-flows and prevent from establishing paths with subzero bandwidth that could be adjusted upon failure occurrence, the traffic volumes that can be thinned and thickened are bounded by a fixed percentage of their nominal path-flows. Summarizing, flows (on existing paths) can be increased due to:

- capacity released on affected paths

- capacity possibly released on some paths of unaffected demands

- protection capacity as a result of network over-dimensioning.

Clearly, the capacity released on routing paths of unaffected demands is limited, because the remaining flows on the routing paths for the unaffected demands must be sufficient to carry the still requested traffic volume. EFR is illustrated by the following example.

Consider a network with 5 nodes $A, B, C, D$, and $E$ and 7 undirected links $(A, B),(A, C),(A, E),(B, D),(B, E),(C, D)$, and $(C, E)$. The unit capacity cost of all seven links equals 1 . We consider two demands $d_{1}$ and $d_{2}$ (each with demand volume equal to 1 ) between nodes $A$ and $E$ for $d_{1}$ and nodes $A$ and $D$ for $d_{2}$. We consider single-link failure scenarios in which each link can fail, but only one at a time. We show below what is the result of the minimum link cost network when EFR is used to recover from single link failures.

The solution requires a capacity of $\frac{1}{3}$ at link $(B, E), \frac{2}{3}$ at link $(C, E)$, and 1 at all other five links; thus, the total dimensioning cost is 6 . In the nominal state, as depicted in Figure 1, demand $d_{1}$ is realized on 3 paths: path $p_{1}=\{(A, E)\}$ with 1 flow value and paths $p_{2}=\{(A, B),(B, E)\}$ and $p_{3}=\{(A, C),(C, E)\}$ 
both with $\frac{1}{3}$ flow value; while $d_{2}$ is routed on 2 paths: $p_{4}=\{(A, B),(B, D)\}$ and $p_{5}=\{(A, C),(C, D)\}$ both with $\frac{2}{3}$ flow value.

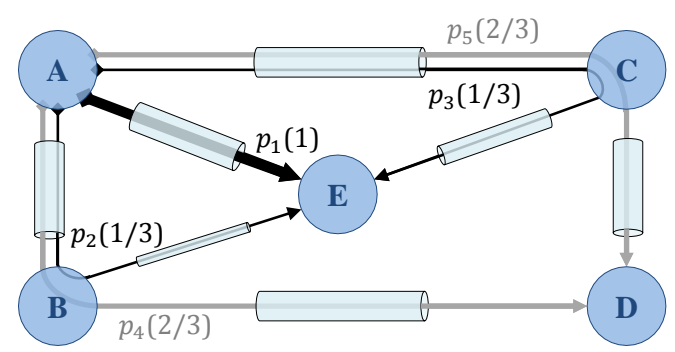

Figure 1: EFR - Nominal State.

Figure 2 represents the failure of link $(A, C)$. Due to the failure, the traffic requirement for $d_{2}$ is no longer met and there is no bandwidth remaining at link $(A, B)$ to increase the traffic of path $p_{4}$. Hence, as demand $d_{1}$ is not affected by the failure, it releases all the bandwidth on path $p_{2}$ (it is possible as still enough bandwidth remains on path $p_{1}$ ). Enough bandwidth is now available to increase the bandwidth on $p_{4}$ by $\frac{1}{3}$; thus, to meet the traffic requirement of $d_{2}$. In this way, all demands are fully operational.

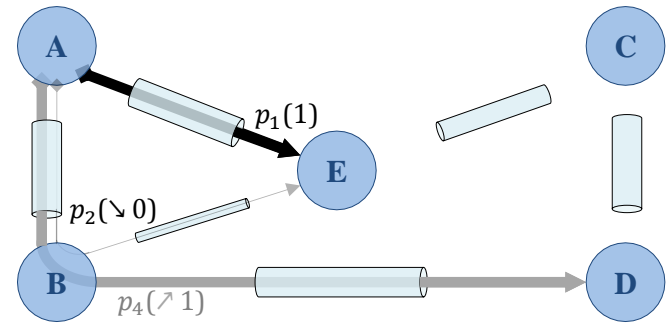

Figure 2: EFR - Failure of link $(A, C)$.

Another failure scenario, presented in Figure 3, is concerned with the failure of link $(A, E)$. The traffic requirement for $d_{1}$ is no longer satisfied, and there is no bandwidth available to increase the traffic on paths $p_{2}$ and $p_{3}$. As demand $d_{2}$ is not affected by the failure, it releases $\frac{1}{3}$ of bandwidth on path $p_{5}$ to make room for path $p_{3}$ thickening. Finally, $d_{1}$ increases the bandwidth of paths $p_{2}$ by $\frac{1}{3}$; thus, all demands recover from the failure. 


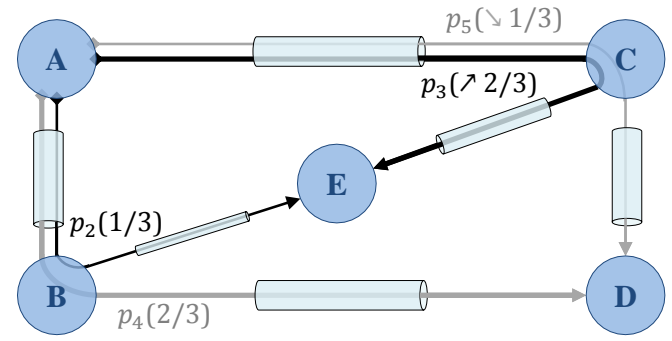

Figure 3: EFR - Failure of link $(A, E)$.

The other failure scenarios are simple of similar to the two examples described above. Failures of links $(B, E)$ and $(C, E)$ do not cause any problem for demand $d_{1}$. The other link failures are close to the failure of $(A, C)$. Note that the dimensioning cost of 6 is also the optimal cost for both GR and RR strategies.

In short, we can say that EFR adds rerouting capabilities to PD, but, unlike RR or GR, seeks to find the capacity needed for rerouting by decreasing some flows on the nominal routing paths of unaffected demands in addition to the capacity released by the affected flows.

\section{Mathematical model of EFR}

The optimization problem considered in this paper is referred to as Elastic Flow Rerouting Problem (EFR-P) and is as follows. The objective is to minimize the total cost of link capacity assuming that in a nominal state, all demand volumes are realized by means of nominal path-flows. When the network is subject to a failure of a link, then the demand volumes, possibly reduced, are realized for the duration of the failure state by the same nominal path-flow, but appropriately thinned or thickened depending on the state of the corresponding demands. The utilized notation is given in Section 3.1, while the detailed formulation of EFR-P is given in Section 3.2. Section 3.3 is devoted to the complexity study of the proposed method.

\subsection{Notation}

The considered network is modeled using a graph $\mathcal{G}=(\mathcal{V}, \mathcal{E})$, undirected or directed, composed of a set of routers $\mathcal{V}$ seen as nodes and a set of links $\mathcal{E}$ seen as arcs or edges. In the sequel, we will always consider undirected graphs unless stated explicitly otherwise. Thus, each link $e \in \mathcal{E}$ represents an unordered pair $\{v, w\}$ of some nodes $v, w \in \mathcal{V}$. We assign to each link $e \in \mathcal{E}$ a non-negative unit capacity $\operatorname{cost} \xi_{e}$, which is a parameter, and a capacity reservation $y_{e}$, which is an optimization variable. The total cost of the network is given by $C=\sum_{e \in \mathcal{E}} \xi_{e} y_{e}$. 
Demands are represented by set $\mathcal{D}$. Each demand $d \in \mathcal{D}$ is associated with an unordered pair of nodes $\{o(d), t(d)\}$. For the sake of simplicity we call $o(d)$ a source node and $t(d)$ a termination node. Volume $h_{d}^{0}$ (a parameter) has to be sent between $o(d)$ and $t(d)$. Notice that demand volumes and link capacities are expressed in the same units. Also, to each demand $d$ we assign a set of admissible paths $\mathcal{P}_{d}$ composed of selected elementary paths between $o(d)$ and $t(d)$ in graph $\mathcal{G}$. Recall that an elementary path does not traverse any node more than once. Paths from $\mathcal{P}_{d}$ are used to realize demand volume $h_{d}^{0}$ by means of nominal path-flows of volume represented by optimization variables $x_{d p}^{0}, p \in \mathcal{P}_{d}$. The given sets of admissible paths define the link-path incidence coefficients $\delta_{\text {edp }}, e \in \mathcal{E}, d \in \mathcal{D}, p \in P_{d}$, where $\delta_{\text {edp }}=1$ if path $p \in \mathcal{P}_{d}$ traverses link $e \in \mathcal{E}$ (i.e., if $e \in p$, treating paths as subsets of links: $p \subseteq E$ ), and $\delta_{e d p}=0$ if path $p \in \mathcal{P}_{d}$ does not traverse link $e$. It is important to note that the sets of admissible paths $\mathcal{P}_{d}, d \in \mathcal{D}$, are parameters in the EFR-P problem formulation.

Network links are subject to totall failures. The set of failure states is denoted by $\mathcal{S}$. Each failure state $s \in \mathcal{S}$ is identified with a set of links $(s \subseteq \mathcal{E})$ that are not operational. The set of paths in $\mathcal{P}_{d}$ that are unaffected by the failure state $s \in \mathcal{S}$, i.e., the set of all paths from $\mathcal{P}_{d}$ that do not contain a link in $s$, is denoted by $\mathcal{P}_{d}^{s}$. Symmetrically, the set of paths that are affected by $s$ is denoted by $\overline{\mathcal{P}}_{d}^{s}$.

A traffic demand $d$ is considered affected by a failure state $s$ if its surviving nominal path-flows are not sufficient to carry the traffic volume requested for this state, i.e., when $\sum_{p \in \mathcal{P}_{d}^{s}} x_{d p}^{0}<h_{d}^{s}$. Otherwise, the demand is unaffected. The status (affected/unaffected) of demand $d \in \mathcal{D}$ in failure state $s \in \mathcal{S}$ is represented, by a binary variable $T_{d}^{s}, d \in \mathcal{D}, s \in \mathcal{S}: T_{d}^{s}=1$ if $d$ is an affected demand in $s$, and $T_{d}^{s}=0$ if $d$ is not affected in $s$.

In failure state $s \in \mathcal{S}$, unaffected demands $d \in \mathcal{D}^{s}$ (resp. affected demands $d \in \overline{\mathcal{D}}^{s}$ ) can only thin (resp. thicken) their unaffected path-flows. This concerns only paths in $\mathcal{P}_{d}^{s}$. The demand volumes associated to a given failure state must be realized for the unaffected demands by means of the surviving nominal pathflows that are appropriately thinned (decreased), and for the affected demandsappropriately thickened (increased). The values by which the nominal pathflows will be thinned (resp. thickened) for the state $s \in \mathcal{S}$ are denoted $u_{d p}^{s}, d \in$ $\mathcal{D}^{s}, p \in \mathcal{P}_{d}^{s}$ (resp. by $v_{d p}^{s}, d \in \overline{\mathcal{D}}^{s}, p \in \mathcal{P}_{d}^{s}$ ). Hence, for a given failure state, all the nominal path-flows of a given demand can be either thinned or thickened. The thinning (resp. thickening) state-dependent path-flows $u_{d p}^{s}$ (resp. $v_{d p}^{s}$ ), $s \in \mathcal{S}, d \in \mathcal{D}, p \in \mathcal{P}_{d}^{s}$, are optimization variables and are bounded by ratio $a \leq 1$ (resp. $b \geq 0$ ) of their nominal values, or by 0 depending on the status of the demand.

\subsection{EFR-P formulation}

Optimization problem EFR-P is given in the non-linear path-flow formulation (1) for a given list of admissible path sets $\mathcal{P}_{d}, d \in \mathcal{D}$. In the formulation, objective (1a) minimizes the total cost of links, i.e., the dimensioning cost. 
Constraints (1b) do not allow nominal link loads exceed nominal link capacities. Constraints (1c) assure that for each demand its paths have jointly sufficient capacity to satisfy the demand volume requested for the nominal state. Next, constraints (1d) force that in each state $s \in \mathcal{S}$, the capacity of each working link $e \in \mathcal{E} \backslash\{s\}$ is not exceeded. Constraints (1e) assure that in each failure state $s \in \mathcal{S}$, the adjusted flows are sufficient to realize the (possibly reduced with respect to $h_{d}^{0}$ ) volume $h_{d}^{s}$ of demand $d \in \mathcal{D}$. Then, constraints (1h) do not allow exceeding the thinning/thickening limits imposed by parameters $a$ and $b$. Constraints (1f) assure that each binary variable $T_{d}^{s}$ takes value $T_{d}^{s}=1$ if the corresponding demand is affected by more than a given $\epsilon>0$ in the failure state $s$, while constraints (1g) deal with unaffected demands. Finally, constraints $(1 \mathrm{~h})-(1 \mathrm{j})$ force variables $x, u$, and $v$ to be correctly defined with respect to affected/unaffected demands and $T$ binary variables.

$$
\begin{array}{r}
\min C=\sum_{e \in \mathcal{E}} \xi_{e} y_{e} \\
\sum_{d \in \mathcal{D}} \sum_{p \in \mathcal{P}_{d}} \delta_{e d p} x_{d p}^{0} \leq y_{e}, \quad e \in \mathcal{E} \\
\sum_{p \in \mathcal{P}_{d}} x_{d p}^{0} \geq h_{d}^{0}, \quad d \in \mathcal{D} \\
\sum_{d \in \mathcal{D}} \sum_{p \in \mathcal{P}_{d}^{s}} \delta_{e d p}\left(x_{d p}^{0}-u_{d p}^{s}+v_{d p}^{s}\right) \leq y_{e}, \quad s \in \mathcal{S}, e \in \mathcal{E} \backslash\{s\} \\
\sum_{p \in \mathcal{P}_{d}^{s}}\left(x_{d p}^{0}-u_{d p}^{s}+v_{d p}^{s}\right) \geq h_{d}^{s}, \quad s \in \mathcal{S}, d \in \mathcal{D} \\
\sum_{p \in \mathcal{P}_{d}^{s}} x_{d p}^{0}+M\left(T_{d}^{s}-1\right) \leq h_{d}^{s}-\epsilon, \quad s \in \mathcal{S}, d \in \mathcal{D} \\
\sum_{p \in \mathcal{P}_{d}^{s}} x_{d p}^{0}+h_{d}^{s} T_{d}^{s} \geq h_{d}^{s}, \quad s \in \mathcal{S}, d \in \mathcal{D} \\
x_{d p}^{0}-a^{-1} u_{d p}^{s}-b^{-1} v_{d p}^{s} \geq 0, \quad s \in \mathcal{S}, d \in \mathcal{D}, p \in \mathcal{P}_{d}^{s} \\
u_{d p}^{s}+M\left(T_{d}^{s}-1\right) \leq 0, \quad s \in \mathcal{S}, d \in \mathcal{D}, p \in \mathcal{P}_{d}^{s} \\
v_{d p}^{s}-M T_{d}^{s} \leq 0, \quad s \in \mathcal{S}, d \in \mathcal{D}, p \in \mathcal{P}_{d}^{s}
\end{array}
$$

$x, y, u, v$ continuous, nonnegative, $T$ binary

Next, we discuss state-dependent constraints (1f),(1g),(1i), and (1j). If $\sum_{p \in \mathcal{P}_{d}^{s}} x_{d p}^{0}<h_{d}^{s}$, demand $d$ is affected by the failure state $s$ and its pathflows can only be thickened, so $u_{d p}^{s}=0$. Otherwise, demand $d$ is not affected and its path-flows can only be thinned, which implies that $v_{d p}^{s}=0$. For this purpose, the constraints use a very large number called big- $M$ coefficient, as well as a very small value $\epsilon$ (constraints (1f)) which, together with $T_{d}^{s}$ binary variables, emulates $I F$-like constraints. Using a large value $M$ and a small value $\epsilon$ typically leads to a high numerical instability. To avoid this issue, constraints (1f),(1g),(1i), and (1j) are modeled in CPLEX, 
the solver utilized in our experiments, as indicator constraints. For instance, the "strictly less than" linear constraints equivalent to (1f) are modeled as $\operatorname{IF}\left(\sum_{p \in \mathcal{P}_{d}^{s}} x_{d p}^{0} \geq h_{d}^{s}\right) T H E N T_{d}^{s}=0$ ELSE $T_{d}^{s}=1$.

\subsection{Complexity discussion}

Formulation (1) is hard to solve exactly by mixed-integer linear solvers for mainly two reasons. First, the number of variables used in the formulation can be very large, since the sets of admissible paths can be of exponential sizes with respect to the size of a network. Second, it contains binary variables and big- $M$ coefficients, which both lead to numerical instability and very poor linear relaxations. All this gives a hint on the practical difficulty and the computational complexity of the EFR problem. Before stating the actual computational complexity let us clarify, in the light of the above formulation, the connection of EFR to other restoration strategies mentioned in Section 1. First, taking $a=0$ and $b=0$ leads to PD strategy (neither thinning nor thickening are possible). Second, for $a=1$ and $b \rightarrow \infty$ one can see that EFR converges to Global Rerouting, while for $a=0$ and $b \rightarrow \infty$ EFR becomes RR strategy. We can easily check that all requirements for each strategy are satisfied. For instance let us consider RR: taking $a=0$ implies that flow thinning is not allowed, while $b \rightarrow \infty$ means that new rerouting paths can be created in practice; this is because the flow on some paths can be enlarged at any finite value starting from practically insignificant flow values. All these special cases have different levels of complexity for the single link failure case: GR and PD fall into the polynomial time complexity class [2], while $\mathrm{RR}$ is shown to be $\mathcal{N} \mathcal{P}$-hard for both the directed [9] and undirected [20] cases. The observation suggests that both problems will exhibit the same $\mathcal{N} \mathcal{P}$ complexity. Not surprisingly, we will use similar arguments to show the $\mathcal{N} \mathcal{P}$-hardness in question. The proof given for EFR is inspired by the RR $\mathcal{N} \mathcal{P}$-hardness proof presented in [9]. The proof is based on a specific network constructed to show that finding an RR solution is equivalent to solving the elementary path problem EL-PATH, which consists in answering the question whether there exists an elementary path going through a fixed link in a directed graph. Problem EL-PATH is $\mathcal{N} \mathcal{P}$-hard, because it can be reduced from 2-DIV-PATH, which itself is $\mathcal{N} \mathcal{P}$-hard [21].

The network instance utilized in the proof is depicted in Figure 4. It assumes a simplified failure scenario involving a single demand and failures of only two links. Considering a single demand only has a particular impact on using EFR the method cannot employ thinning as there is no interest in considering cases when the demand is unaffected. Hence, in the following, we consider the EFR problem with $a$ set to 0 and $b$ set to a large but finite value, thus the nominal flows cannot be decreased, but they can be increased and multiplied by $b+1$. Then, bearing in mind that we are looking for solutions involving only elementary routing paths, the following proposition can be proven.

Proposition 1. EFR problem is $\mathcal{N} \mathcal{P}$-hard already for the case with two failing links and one demand. 
Proof. The proof is deduced from the problem of existence of an elementary path in a directed graph traversing a given link. Let this problem be denoted by EL-PATH. As discussed in [9], EL-PATH (the decision problem consisting in answering whether there exists an elementary path going through a given link in a directed graph) is $\mathcal{N} \mathcal{P}$-hard.

The upper left part of Figure 4 represents an instance of EL-PATH, asking for finding whether there exists an elementary path from $v$ to $w$ traversing a given link $(a, b)$ in a directed graph represented by the oval. The main part of the figure shows how this instance of EL-PATH can be reduced to an instance of the considered case of EFR with one demand $d$ from node $o$ to node $t$ (with volume $h_{d}=1$ ) and two failing links $f_{1}$ and $f_{2}$.

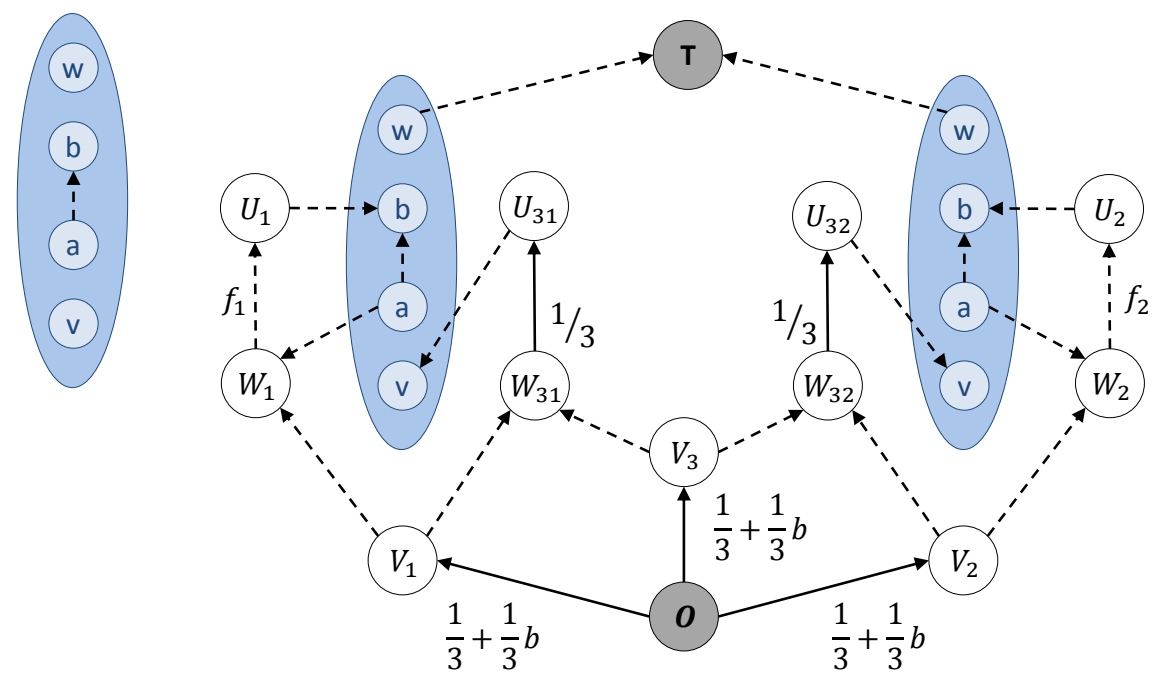

Figure 4: Illustration of the proof.

In the main graph, the links depicted by solid lines have large unit costs $\xi$, while unit costs of the links drawn as dotted lines are negligible with respect to $\xi$. The two ovals in the main graph are the copies of the oval in the upper left part. Unit costs of all possible links in these two ovals are also negligible as compared to $\xi$. Note that the nodes in both ovals in the main graph have the same names, but this should not lead to a confusion.

We first show that the objective function value $C$ of any feasible solution of EFR must be greater than or equal to $\frac{5}{3} \xi$. To demonstrate this, consider the following cuts involving the expensive links, i.e., links $\left(o, v_{1}\right),\left(o, v_{2}\right),\left(o, v_{3}\right)$, $\left(w_{31}, u_{31}\right)$, and $\left(w_{32}, u_{32}\right)$ :

- one cut for the normal state: $\left\{\left(o, v_{1}\right),\left(o, v_{2}\right),\left(o, v_{3}\right)\right\}$

- first cut for failure of $f_{1}:\left\{\left(w_{31}, u_{31}\right),\left(w_{32}, u_{32}\right),\left(o, v_{2}\right)\right\}$

- second cut for failure of $f_{1}:\left\{\left(w_{31}, u_{31}\right),\left(o, v_{2}\right),\left(o, v_{3}\right)\right\}$ 
- first cut for failure of $f_{2}:\left\{\left(o, v_{1}\right),\left(o, v_{3}\right),\left(w_{32}, u_{32}\right)\right\}$

- second cut for failure of $f_{2}:\left\{\left(w_{31}, u_{31}\right),\left(w_{32}, u_{32}\right),\left(o, v_{1}\right)\right\}$.

The capacity of each of these five cuts must be greater than or equal to the requested volume $h_{d}=1$. Hence, by summing up the inequalities expressing these conditions for all five cuts we see that the sum of the capacity reservations of the five expensive links must be at least $\frac{5}{3}$. As each of these links has the unit cost $\xi$, we conclude that $C \geq \frac{5}{3} \xi$.

Now we will establish bounds of optimal solutions of EFR in the case when an elementary path from $v$ to $w$ traversing link $(a, b)$ in the oval exists (Case $\mathbf{1}$ ), and in the case when it does not exist (Case 2).

Case 1: Suppose that the oval contains an elementary path $P$ from $v$ to $w$ traversing link $(a, b)$. Then, there exists a solution that achieves the cost $C^{0}=\frac{5}{3} \xi+\frac{1}{b} \xi$. In this solution, the flows in the normal state are as follows:

- $\frac{1}{3}$ : path $o-v_{1}-w_{1}-u_{1}-(b-w$ part of $P)-t$

- $\frac{1}{6}$ : path $o-v_{3}-w_{31}-u_{31}-v-(v-a$ part of $P)-w_{1}-u_{1}-b-$ $(b-w$ part of $P)-t$

- $\frac{1}{6}$ : path $o-v_{3}-w_{32}-u_{32}-v-(v-a$ part of $P)-w_{2}-u_{2}-b-$ $(b-w$ part of $P)-t$

- $\frac{1}{3}$ : path $o-v_{2}-w_{2}-u_{2}-(b-w$ part of $P)-t$.

- $\frac{1}{6 b}:$ patho $-v_{3}-w_{32}-u_{32}-v-P-t$

- $\frac{1}{3 b}$ : path $o-v_{1}-w_{31}-u_{31}-v-P-t$.

- $\frac{1}{6 b}$ : path $o-v_{3}-w_{31}-u_{31}-v-P-t$

- $\frac{1}{3 b}$ : path $o-v_{1}-w_{32}-u_{32}-v-P-t$.

When link $f_{1}$ fails, flow on $o-v_{3}-w_{32}-u_{32}-v-P-t$ is enlarged to reach $\frac{1}{6}$ and flow on $o-v_{1}-w_{31}-u_{31}-v-P-t$ is thickened to $\frac{1}{3}$. When link $f_{2}$ fails, the third and the fourth normal flows are rerouted in a symmetric way by enlarging respectively the flows on path $o-v_{3}-w_{31}-u_{31}-v-P-t$ and path $o-v_{1}-w_{32}-u_{32}-v-P-t$. The resulting capacity reservations of the expensive, solid-line links are all equal to $\frac{1}{3}$ (for links $w_{31}-v_{31}$ and $w_{32}-v_{32}$ ), and $\left(\frac{1}{3}+\frac{1}{3 b}\right)$ for the three other links (respectively $o-V-1, O-v-2$, and $\left.o-v_{3}\right)$ as depicted in Figure 4. The resulting capacity is $\left(\frac{5}{3}+\frac{1}{b}\right) \xi$ and for $b=36$ it gives a cost of $\frac{61}{36} \xi$. 
Case 2: Now assume that there is no elementary path from $v$ to $w$ through $(a, b)$ in the oval. We will show that in this case the cost $C^{0}$ of an optimal solution of EFR must be greater than or equal to $\frac{7}{4} \xi$. Indeed, it has been shown in [9] that any solution for RR in the considered network is necessarily greater than or equal to $\frac{7}{4} \xi$, which is greater than $\frac{61}{36} \xi$. This holds also for EFR as for $a=0$ all EFR solutions are necessarily solutions of RR, so EFR cannot do better than RR.

To summarize, solving the constructed instance of EFR for the optimal cost $C^{0}$ yields a solution of EL-PATH: an elementary path $P$ in question exists if $C^{0} \leq \frac{61}{36} \xi$, and does not exist when $C^{0} \geq \frac{7}{4} \xi$. In this way, EL-PATH is reduced to EFR and hence EFR is $\mathcal{N} \mathcal{P}$-hard.

\section{Heuristic method}

As remarked in the previous section, formulation (1) combines the big- $M$ constraints with a large number $O(|\mathcal{D}||\mathcal{S}|)$ of binary variables, and produces largescale integer-programming problems which happen to be intractable even for moderate size networks.

Solving the EFR-P requires solving a combinatorial optimization problem over the combinations of affected/unaffected demands sets for each failure. Additionally, it requires applying path generation in the solution process. In consequence, the exact solution of EFR-P would require branch-and-price algorithms. Obviously, solving formulation (1) with binary variables $T_{d}^{s}$ set to fixed values representing a certain choice of affected/unaffected demands would give an upper bound for the optimal solution of EFR-P. In fact, as we will see below, the optimization problem behind such upper bound solutions is much simpler to solve than EFR-P itself.

Hence, a natural heuristic approach solving EFR-P would be to sequentially approach a set of problems with the unaffected/affected demands gradually fixed in each iteration. Such a mindset leads to a greedy heuristic approach described in Section 4.1. Next, the problem solved at each iteration, called EFR-PF (EFR-P fixed), is presented in detail in Section 4.2.

\subsection{Description of the heuristic}

In this section, we present the greedy heuristic method used to solve the EFR$\mathrm{P}$ optimization problem. In each iteration, the heuristic creates and solves an instance of EFR-PF (version of EFR-P (1), in which all $T_{d}^{s}, d \in \mathcal{D}, s \in \mathcal{S}$, are fixed to 0 or 1), and then adjusts the states (affected/unaffected) of the demands. The process is repeated until no improvement is achieved - more precisely, it is finished when all demands' states remain unchanged.

The algorithm starts with an empty set of unaffected demands for each failure state, and with some initial admissible path-sets. Thus, at the beginning all demands are considered as potentially affected. After solving the corresponding LP, we know exactly which demands are affected and non-affected in the 
current solution $x$. This gives a hint how to update the unaffected demands for the next iteration - the set of unaffected demands is extended with the demands that are not affected in the obtained solution $x$. In this way, the new unaffected demands can contribute to the restoration process by potentially releasing some flow on their paths. For the next iteration, the EFR-PF instance is extended with the constraints that characterize the demands recently moved to the set of unaffected demands.

In consequence, any feasible solution will always keep these demands unaffected because of the recently added constraints, which are not subject to removal in the subsequent steps of the iteration process. Therefore, at each iteration, the obtained solution $x$ can only extend the set of unaffected demands and no demands that have been previously made unaffected can become affected. The process of updating the sets of unaffected demands continues until a solution to LP returns the same set of unaffected demands in two consecutive iterations. This is the key idea of the method used in Heuristic EFR-H detailed in Algorithm 1 below.

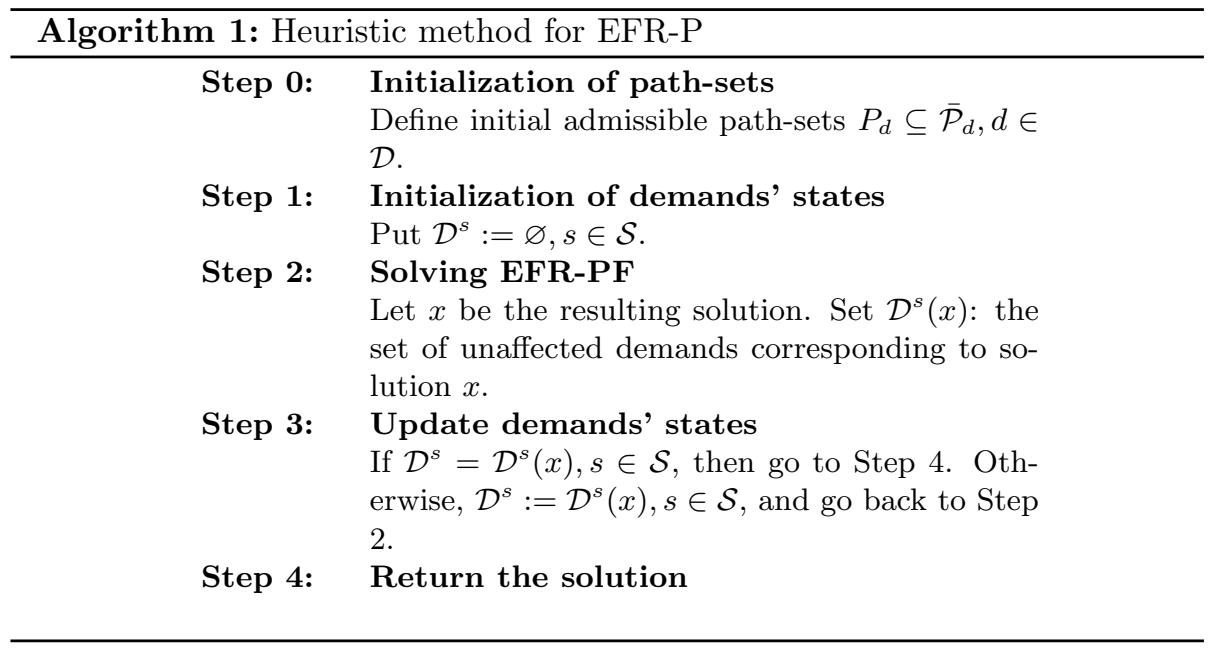

We can easily deduce that the algorithm will always stop, because once a demand is considered unaffected it will remain unaffected for all consecutive iterations. On the other hand, affected demands can change their states and become unaffected. Hence, the process will necessarily stop since the set of unaffected demands cannot be extended to infinity, and the algorithm ends when we obtain the same set of unaffected demands for two consecutive iterations.

One of the disadvantages of the presented algorithm is its fast convergence to local minima. If a large set of demands becomes unaffected in a single iteration, the heuristic may not be able to find high quality solutions. In order to alleviate this issue, the number of demands that can become unaffected in a failure state $s$ in a single iteration is limited to $M A X_{d}$. If $M A X_{d}$ takes a very low value, the heuristic will slowly converge, will explore a lot more scenarios, but the 
computation time will be much longer. In contrast, if $M A X_{d}$ takes a high value, the computations will be fast, but the algorithm will quickly converge to a local minimum that can be very far from the true optimum. The most reasonable value of $M A X_{d}$ depends on a network instance. However, we notice that $M A X_{d}=5$ represents a good compromise between the computation time and the solution quality. Still, observe that the presented method is not exact; thus, obtained solutions are in general suboptimal although we have already achieved almost optimal results in the majority of tests (look at Section 7).

\subsection{Formulation of EFR-PF problem}

Let us now look in detail how the EFR-PF problem used in each iteration of the above algorithm is formally defined. We first introduce additional notation by defining two sets: $\mathcal{E}(d)=\left\{s \in \mathcal{S}: d \in \mathcal{D}^{s}\right\}$ is the set of links whose failures do not affect demand $d$, and $\overline{\mathcal{E}}(d)=\left\{s \in \mathcal{S}: d \in \overline{\mathcal{D}}^{s}\right\}$ is the set of links whose failures affect demand $d$. Note that in the EFR-PF problem the sets $\mathcal{D}^{s}, s \in \mathcal{S}$ are predefined. Then, a linear programming formulation for EFR-PF, denoted by $P_{F}\left(\mathcal{D}^{s}, \mathcal{P}\right)$ and given in (2), can be obtained from (1) by fixing the variables accordingly.

$$
\begin{array}{r}
\min C=\sum_{e \in \mathcal{E}} \xi_{e} y_{e} \\
{\left[\pi_{e}^{0}\right] \sum_{d \in \mathcal{D}} \sum_{p \in \mathcal{P}_{d}} \delta_{e d p} x_{d p}^{0} \leq y_{e}, \quad e \in \mathcal{E}} \\
{\left[\lambda_{d}^{0}\right] \sum_{p \in \mathcal{P}_{d}} x_{d p}^{0} \geq h_{d}^{0}, \quad d \in \mathcal{D}} \\
{\left[\pi_{e}^{s}\right] \sum_{d \in \mathcal{D}} \sum_{p \in \mathcal{P}_{d}^{s}} \delta_{e d p} x_{d p}^{0}-\sum_{d \in \mathcal{D}^{s}} \sum_{p \in \mathcal{P}_{d}^{s}} \delta_{e d p} u_{d p}^{s}+\sum_{d \in \overline{\mathcal{D}}^{s}} \sum_{p \in \mathcal{P}_{d}^{s}} \delta_{e d p} v_{d p}^{s} \leq y_{e}} \\
s \in \mathcal{S}, e \in \mathcal{E} \backslash\{s\} \\
\sum_{p \in \mathcal{P}_{d}^{s}}\left(x_{d p}^{0}-u_{d p}^{s}\right) \geq h_{d}^{s}, \quad d \in \mathcal{D}^{s}, s \in \mathcal{E}(d) \\
\sum_{p \in \mathcal{P}_{d}^{s}}\left(x_{d p}^{0}+v_{d p}^{s}\right) \geq h_{d}^{s}, \quad d \in \overline{\mathcal{D}}^{s}, s \in \overline{\mathcal{E}}(d) \\
{\left[\sigma_{d p}^{s}\right] \quad u_{d p}^{s} \leq a x_{d p}^{0}, \quad s \in \mathcal{E}(d), p \in \mathcal{P}_{d}^{s}} \\
{\left[\sigma_{d p}^{s}\right] \quad v_{d p}^{s} \leq b x_{d p}^{0}, \quad s \in \overline{\mathcal{E}}(d), p \in \mathcal{P}_{d}^{s}} \\
x, u, z, y \text { continuous, nonnegative }
\end{array}
$$

In formulation (2), objective (2a) minimizes the total cost of links, and (2b)(2f) are similar to (1b)-(1e) defined for EFR-P. Constraint (1e) is split in two constraints (2e) and (2f). Then, constraints $(2 \mathrm{~g})$ do not allow releasing more than fraction $a$ of the nominal flow assigned to a path, and constraints $(2 \mathrm{~h})$ ensure that flow increase on a disrupted path by more than fraction $b$ of the 
nominal flow is not permitted. The values of $a$ and $b$ are fixed parameters with $0 \leq a \leq 1, b \geq 0$. In the formulation, the quantities in brackets to the left are dual variables associated with constraints. All these variables are, by assumption, non-negative.

The condition stipulating that surviving flows of the affected demands are not sufficient to satisfy the required demand volume can be expressed as:

$$
\sum_{p \in \mathcal{P}_{d}^{s}} x_{d p}^{0} \leq h_{d}^{s}-\epsilon, s \in \mathcal{S}, d \in \overline{\mathcal{D}}^{s},
$$

for a very small $\epsilon>0$. Nevertheless, we have deliberately chosen not to introduce this constraint into the EFR-PF formulation $(2)^{1}$. In practice, the EFR-PF formulation (2) solved in the first iteration of the algorithm does not contain constraints (2e), because $\mathcal{D}^{s}$ is initally set to $\emptyset$. Having a solution, each demand $d \in D$ is checked if it satisfies (3). If constraint (3) is not satisfied for a particular demand, the demand is added to set $\mathcal{D}^{s}$. At this stage, the obtained solution $x$ remains feasible for the new LP problem. Thus, the heuristic generates a sequence of approximate solutions that are improving in time.

Although EFR-PF is an LP, the problem is still not easy to solve because of the possibly exponential numbers of path-flow variables $x, u$, and $z$. To avoid the non-compact nature of the formulation, paths are generated on the fly using Path Generation (PG) applied to formulation (2). Unfortunately, PG turns out to be a difficult problem as it is shown in the following subsections.

\subsection{Dual problem}

Let us consider the problem dual to problem (2) for a given set of admissible paths $\mathcal{P}$. The dual, denoted by $D(\mathcal{P})$, is as follows (for the derivation see for example [1]):

$$
\begin{array}{r}
\max W=\sum_{d \in \mathcal{D}}\left(h_{d}^{0} \lambda_{d}^{0}+\sum_{s \in \mathcal{S}} h_{d}^{s} \lambda_{d}^{s}\right) \\
\pi_{e}^{0}+\sum_{s \in \mathcal{S} \backslash\{e\}} \pi_{e}^{s}=\xi_{e}, \quad e \in \mathcal{E} \\
\lambda_{d}^{0}+\sum_{s \in \mathcal{E}(d) \backslash p}\left(\lambda_{d}^{s}+a \sigma_{d p}^{s}\right)+\sum_{s \in \overline{\mathcal{E}}(d) \backslash p}\left(\lambda_{d}^{s}+b \sigma_{d p}^{s}\right) \leq \sum_{s \in \mathcal{S} \backslash p}|p|^{s}+|p|^{0}, \\
d \in \mathcal{D}, p \in \mathcal{P}_{d} \\
\lambda_{d}^{s}+\sigma_{d p}^{s} \geq|p|^{s}, \quad d \in \mathcal{D}, p \in \mathcal{P}_{d}, s \in \mathcal{E}(d) \backslash p \\
\lambda_{d}^{s}-\sigma_{d p}^{s} \leq|p|^{s}, \quad d \in \mathcal{D}, p \in \mathcal{P}_{d}, s \in \overline{\mathcal{E}}(d) \backslash p \\
\lambda, \pi, \sigma \text { continuous, nonnegative }
\end{array}
$$

\footnotetext{
${ }^{1}$ The idea behind this relies on the way the above heuristic works: we do not need to constrain a solution obtained at each step of the heuristic to a fixed set of affected/unaffected demands, but only need to prevent the set of unaffected demands from shrinking.
} 
where, for a given path $p \in \mathcal{P}$, we have used the notation $|p|^{0}=\sum_{e \in p} \pi_{e}^{0}$ and $|p|^{s}=\sum_{e \in p} \pi_{e}^{s}$.

We notice that the optimal solution of (4) must give for $d \in \mathcal{D}, p \in \mathcal{P}_{d}, s \in$ $\mathcal{E}(d) \backslash p$ :

$$
\sigma_{d p}^{s}= \begin{cases}0, & \text { if }|p|^{s} \leq \lambda_{d}^{s} \\ |p|^{s}-\lambda_{d}^{s}, & \text { if }|p|^{s}>\lambda_{d}^{s}\end{cases}
$$

and for $d \in \mathcal{D}, p \in \mathcal{P}_{d}, s \in \overline{\mathcal{E}}(d) \backslash p$ :

$$
\sigma_{d p}^{s}= \begin{cases}|p|^{s}-\lambda_{d}^{s}, & \text { if }|p|^{s}<\lambda_{d}^{s} \\ 0, & \text { if }|p|^{s} \geq \lambda_{d}^{s}\end{cases}
$$

Observe that if we replace $\sigma_{d p}^{s}$ with these values, formulation (4) and formulation (9) given below have the same sets of feasible variables $\pi$ and $\lambda$. We introduce some additional notation. For $d \in \mathcal{D}, p \in \mathcal{P}_{d}$ :

$$
\mathcal{F}_{d}(p)=\left\{s \in \mathcal{E}(d) \backslash p:|p|^{s}>\lambda_{d}^{s}\right\}
$$

Symmetrically, for $d \in \mathcal{D}, p \in \mathcal{P}_{d}$ :

$$
\overline{\mathcal{F}}_{d}(p)=\left\{s \in \overline{\mathcal{E}}(d) \backslash p:|p|^{s}<\lambda_{d}^{s}\right\}
$$

Considering (5)-(8), the transformed formulation of the dual problem is as follows:

$$
\begin{array}{r}
\max W=\sum_{d \in \mathcal{D}}\left(h_{d}^{0} \lambda_{d}^{0}+\sum_{s \in \mathcal{S}} h_{d}^{s} \lambda_{d}^{s}\right) \\
\pi_{e}^{0}+\sum_{s \in \mathcal{S} \backslash\{e\}} \pi_{e}^{s}=\xi_{e}, \quad e \in \mathcal{E} \\
\lambda_{d}^{0}+a \sum_{s \in \mathcal{F}_{d}(p)} \Delta_{d p}^{s}+b \sum_{s \in \overline{\mathcal{F}}_{d}(p)} \Delta_{d p}^{s}-\sum_{s \in \mathcal{S} \backslash p} \Delta_{d p}^{s} \leq|p|^{0}, \quad d \in \mathcal{D}, p \in \mathcal{P}_{d} \\
\lambda, \pi \text { continuous, nonnegative }
\end{array}
$$

with $\Delta_{d p}^{s}=|p|^{s}-\lambda_{d}^{s}, d \in \mathcal{D}, p \in \mathcal{P}_{d}, s \in \mathcal{S} \backslash p$.

\subsection{Pricing problem}

Let $\overline{\mathcal{P}}_{d}$ denote the set of all paths in the network graph between $o(d)$ and $t(d)$. The essence of the pricing problem related to the dual problem (9) formulated for a given (limited) admissible path-sets $\mathcal{P}_{d}, d \in \mathcal{D}$, is to check whether for at least one demand $d \in \mathcal{D}$ there exists a path $q \in \overline{\mathcal{P}}_{d} \backslash \mathcal{P}_{d}$ (i.e., path $q$ outside the current admissible path-set $\mathcal{P}_{d}$ ), for which (9c) cannot be satisfied for any set of non-negative dual variables $\sigma_{d q}^{s}, s \in \overline{\mathcal{E}}(d) \backslash q$. In fact, this is the case if and only if:

$$
\Delta_{d q}^{0}+\sum_{s \in \mathcal{S} \backslash q} \Delta_{d q}^{s}-a \sum_{s \in \mathcal{F}_{d}(q)} \Delta_{d q}^{s}-b \sum_{s \in \overline{\mathcal{F}}_{d}(q)} \Delta_{d q}^{s}<0
$$


with $\Delta_{d q}^{0}=|q|^{0}-\lambda_{d}^{0}, d \in \mathcal{D}$.

The Pricing Problem (PP) for a given $\pi, \lambda$ and a given demand $d \in \mathcal{D}$ is as follows:

$$
\text { minimize }\|q\| \text { over } q \in \hat{\mathcal{P}}_{d}
$$

where

$$
\|q\|=\Delta_{d q}^{0}+\sum_{s \in \mathcal{S} \backslash q} \Delta_{d q}^{s}-a \sum_{s \in \mathcal{F}_{d}(q)} \Delta_{d q}^{s}-b \sum_{s \in \overline{\mathcal{F}}_{d}(q)} \Delta_{d q}^{s} .
$$

Observe that if $\|q\|<0$ for a path $q$ satisfying (11), then the current optimal dual solution $\pi, \lambda$ violates dual constraints (9c) for path $q$. Moreover, the violation is maximal over all paths $p \in \hat{\mathcal{P}}_{d}$.

Finally, we note that PP is difficult. In particular, the term $\sum_{s \in \mathcal{S} \backslash q} \sum_{e \in p} \pi_{e}^{s}$ included in $\sum_{s \in \mathcal{S} \backslash q} \Delta_{d q}^{s}$ is difficult to deal with. This problem is also known in the literature as the quadratic shortest path problem, since its cost depends not only on the arcs included in the path, but also on the other arcs. Furthermore, it is encountered in the pricing problem of $\mathrm{RR}$ known to be $\mathcal{N} \mathcal{P}$-hard, see [6-9] and a survey on path generation [2]. In our experiments, we have incorporated all potential paths in the initial path set.

\section{$5 \quad$ EFR - multiple partial link failures}

In this section, we present an extension of EFR, referred to as EFR $\mathrm{PLF}_{\text {, in- }}$ tended to handle partial link failures. It is among the first attempts to address flow protection against multiple partial failures in an implementable way. The approach is based on two (re)routing strategies: Flow-Thinning and EFR.

Flow-Thinning (FT) [16] is a protection strategy resilient to multiple partial link failures. The main idea behind FT is to, when a partial failure occurs, adjust the nominal path-flows by thinning them down so the flows are not rerouted.

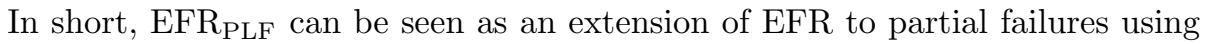
the features of FT. The approach works as follows. When a link partially fails, the affected nominal path-flows can only be thinned following the idea of FT. Next, flow adjustments are made according to EFR. The above implies that EFR $_{\text {PLF }}$ becomes pure EFR when applied to total link failures. In this section, we present the optimization problem (referred to as EFR $\mathrm{PLF}-\mathrm{P}$ ) associated to the EFR $_{\text {PLF }}$ approach discussed above.

Network links are subject to capacity variations that we consider as partial failures. The set of failure states is still denoted by $\mathcal{S}$. Each failure state $s \in \mathcal{S}$ is identified with a set of links $(s \subseteq \mathcal{E})$ that cannot operate at full capacity. As for EFR, the set of paths in $\mathcal{P}_{d}$ that are unaffected by the failure state $s \in \mathcal{S}$, i.e., the set of all paths from $\mathcal{P}_{d}$ that do not contain any link in $s$, is denoted by $\mathcal{P}_{d}^{s}$. Symmetrically, the set of paths that are affected by $s$ is denoted by $\overline{\mathcal{P}}_{d}^{s}$. The nominal path-flow $x_{d p}^{0}$ affected by $s\left(p \in \overline{\mathcal{P}}_{d}^{s}\right)$ can only be thinned-they are thinned to $z_{d p}^{s}$, which are optimization variables. A traffic demand $d$ is said to be affected by a failure state $s$ if its surviving nominal path-flows (thinned or not) are not sufficient to carry the traffic volume assumed for this state, i.e., 
when $\sum_{p \in \overline{\mathcal{P}}_{d}^{s}} z_{d p}^{s}+\sum_{p \in \mathcal{P}_{d}^{s}} x_{d p}^{0}<h_{d}^{s}$. Otherwise, the demand is unaffected. The state (affected/unaffected) of demand $d \in \mathcal{D}$ in failure state $s \in \mathcal{S}$ is represented, as for EFR, by a binary variable $T_{d}^{s}, d \in \mathcal{D}, s \in \mathcal{S}: T_{d}^{s}=1$ if $d$ is an affected demand in $s$, and $T_{d}^{s}=0$ if $d$ is not affected in $s$. The remaining variables are as in EFR-P and the process is based on the same idea as for EFR. In failure state $s \in \mathcal{S}$, unaffected demands $d \in \mathcal{D}^{s}$ (resp. affected demands $d \in \overline{\mathcal{D}}^{s}$ ) can only thin (resp. thicken) their unaffected path-flows. This will concern only paths in $\mathcal{P}_{d}^{s}$. The values by which the nominal flows are thinned (resp. thickened) are denoted by $u_{d p}^{s}$ (resp. $v_{d p}^{s}$ ) and bounded by ratio $a \leq 1$ (resp. $b \geq 0$ ) of their nominal values, or by 0 depending on the state of the demand. The mathematical formulation of $\mathrm{EFR}_{\mathrm{PLF}}$, inspired from EFR, is given below:

$$
\begin{array}{r}
\min C=\sum_{e \in \mathcal{E}} \xi_{e} y_{e} \\
\sum_{d \in \mathcal{D}} \sum_{p \in \mathcal{P}_{d}} \delta_{e d p} x_{d p}^{0} \leq y_{e}, \quad e \in \mathcal{E} \\
\sum_{p \in \mathcal{P}_{d}} x_{d p}^{0} \geq h_{d}^{0}, \quad d \in \mathcal{D} \\
\sum_{d \in \mathcal{D}}\left(\sum_{p \in \mathcal{P}_{d}^{s}} \delta_{e d p}\left(x_{d p}^{0}-u_{d p}^{s}+v_{d p}^{s}\right)+\sum_{p \in \overline{\mathcal{P}}_{d}^{s}} \delta_{e d p} z_{d p}^{s}\right) \leq \alpha_{e}^{s} y_{e}, s \in \mathcal{S}, e \in \mathcal{E} \\
\sum_{p \in \mathcal{P}_{d}^{s}} \delta_{e d p}\left(x_{d p}^{0}-u_{d p}^{s}+v_{d p}^{s}\right)+\sum_{p \in \overline{\mathcal{P}}_{d}^{s}} \delta_{e d p} z_{d p}^{s} \geq h_{d}^{s}, \quad s \in \mathcal{S}, d \in \mathcal{D} \\
\sum_{p \in \overline{\mathcal{P}}_{d}^{s}} z_{d p}^{s}+\sum_{p \in \mathcal{P}_{d}^{s}} x_{d p}^{0}+M\left(T_{d}^{s}-1\right) \leq h_{d}^{s}-\epsilon, \quad s \in \mathcal{S}, d \in \mathcal{D} \\
\sum_{p \in \overline{\mathcal{P}}_{d}^{s}} z_{d p}^{s}+\sum_{p \in \mathcal{P}_{d}^{s}} x_{d p}^{0}+h_{d}^{s} T_{d}^{s} \geq h_{d}^{s}, \quad s \in \mathcal{S}, d \in \mathcal{D} \\
0 \leq u_{d p}^{s} \leq a x_{d p}^{0}, \quad s \in \mathcal{S}, d \in \mathcal{D}, p \in \mathcal{P}_{d}^{s} \\
0 \leq v_{d p}^{s} \leq b x_{d p}^{0}, \quad s \in \mathcal{S}, d \in \mathcal{D}, p \in \mathcal{P}_{d}^{s} \\
0 \leq z_{d p}^{s} \leq x_{d p}^{0}, \quad d \in \mathcal{D}, s \in \mathcal{S}, p \in \overline{\mathcal{P}}_{d}^{s} \\
u_{d p}^{s}+M\left(T_{d}^{s}-1\right) \leq 0, \quad s \in \mathcal{S}, d \in \mathcal{D}, p \in \mathcal{P}_{d}^{s} \\
v_{d p}^{s}-M T_{d}^{s} \leq 0, \quad s \in \mathcal{S}, d \in \mathcal{D}, p \in \mathcal{P}_{d}^{s} \\
x, y, z, u, v \operatorname{continuous,} \text { nonnegative, } T \text { binary }
\end{array}
$$

where the objective function is to minimize the total cost of the network. Constraints (12b) do not allow the nominal link loads to exceed the nominal link capacities. Constraints (12c) ensure that the nominal flows are sufficient to satisfy each demand $d \in \mathcal{D}$.

Constraints (12e) assure that in each failure state $s \in \mathcal{S}$ the adjusted flows are sufficient to realize the volume of each demand $d \in \mathcal{D}$ and constraints (12d) verifie that the surviving capacity of each link $e \in \mathcal{E}$ is not exceeded. Constraints (12f) - (12g) are related to the states of demands. They restrict $T_{d}^{s}$ values to be 
equal to 1 for an affected demand and 0 otherwise. Note that (12f) is handled by CPLEX MIP solver in the same way as (1f) in Section 3. Constraints (12h) - (12l) express bound constraints for variables $u, v$, and $z$.

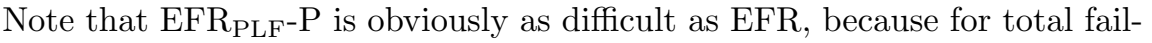
ures it reduces to EFR. This statement holds since flow thinning can be applied only for partial failures - it completely disappears when the link failure cuts the traffic entirely (total failure). Therefore, the already demonstrated $\mathcal{N} \mathcal{P}$ hardness of EFR immediately implies the following corollary.

Corollary 1. The EFR $R_{P L F}$ problem represented by (12) is $\mathcal{N} \mathcal{P}$-hard.

\section{Practical applications of EFR and EFR PLF}

The first part of the study presented in this paper deals with total link failures. The importance of such problems is very well established, as such total link failure problems arise in almost all telecommunication networks. Therefore, in this section, we focus on justifying the importance of problems that deal with partial link failures. We concentrate on broadband wireless communications networks in our explanations, as these networks hold great promise for providing private high-speed data connections by means of microwave radio $[11,15]$ or free space optical (FSO) transmission $[17,19]$. Both microwave and optical transmission refer to terrestrial point-to-point digital communications, usually employing highly directional antennas within the line-of-sight (LOS). What is important, the two technologies operate at unlicensed frequency bands, contrary to radio communications based for instance on LTE. Moreover, both microwave and FSO are sensitive to interferences - the feature we utilize in our optimization model. Despite recent advances in optimization of fixed broadband wireless networks, a variety of questions in this area have not been addressed yet. Particularly, capacity planning in fixed wireless networks is quite different from that in wired networks. In fact, environmental conditions, especially weather, play an important role since they can introduce instantaneous variations into the communication channel, likely leading to outage events which can be modeled as multiple partial link failures. As a common practice, operators tend to highly over-provision bandwidth during network planning to avoid traffic bottlenecks under adverse scenarios (when the performance of some links deteriorates). This approach, however, incurs additional costs that do not result in resource- and cost-efficient networks, leading to an inefficient use of the radio spectrum.

Below we discuss an issue of how to manage partial failures in fixed wireless networks. Even though FSO and microwave radio have a lot in common, in this section, we will focus on microwave radio communications. Radio frequency spectrum is a limited resource regulated worldwide by the International Telecommunications Union (ITU). In conjunction with the ITU regulations, national legislation instruments establish the availability of frequency bands for specific applications and the procedures to use licenses. A license (assignment) is an authorization given by administration for a radio station to use a radio 
frequency under specified conditions. In most cases (also assumed in this paper), the price of frequency spectrum for a single microwave link is a function of the amount of spectrum (bandwidth) in $\mathrm{MHz}$ associated with the license.

Commonly, to support broadband applications, modern microwave systems use quadrature amplitude modulation (QAM). An $m$-QAM scheme utilizes $m$ combinations of amplitude and phase, each one representing an $n$-bit pattern called a symbol (with $n=\log _{2} m$ and integer). Given the channel bandwidth $B$ and the $m$-QAM scheme in use, we can approximate the channel capacity $C$ by:

$$
C[M b / s]=n B[M H z] .
$$

The $m$-QAM schemes with high values of $m$ assure bandwidth efficiency, but are more susceptible to errors due to channel impairments. As the modulation scheme changes to accommodate higher data rates, the signal-to-noise ratio (SNR) requirement increases to preserve the bit error rate (BER).

Table 1: Bandwidth efficiency, SNR requirement, and capacity.

\begin{tabular}{lllll}
\hline $\begin{array}{l}\text { Modulation } \\
\text { scheme }\end{array}$ & $\begin{array}{l}\text { Bandwidth } \\
\text { efficiency }\end{array}$ & $\begin{array}{l}\text { SNR } \\
\text { require- } \\
\text { ment }\end{array}$ & $\begin{array}{l}\text { Capacity } \\
\text { for } \\
7 \mathrm{MHz}\end{array}$ & $\begin{array}{l}\text { Capacity } \\
\text { for } 28 \mathrm{MHz}\end{array}$ \\
\hline \hline QPSK & $2 \mathrm{bps} / \mathrm{Hz}$ & $14.21 \mathrm{~dB}$ & $14 \mathrm{Mbps}$ & $56 \mathrm{Mbps}$ \\
16-QAM & $4 \mathrm{bps} / \mathrm{Hz}$ & $21.02 \mathrm{~dB}$ & $28 \mathrm{Mbps}$ & $112 \mathrm{Mbps}$ \\
32-QAM & $5 \mathrm{bps} / \mathrm{Hz}$ & $25.24 \mathrm{~dB}$ & $35 \mathrm{Mbps}$ & $140 \mathrm{Mbps}$ \\
64-QAM & $6 \mathrm{bps} / \mathrm{Hz}$ & $27.45 \mathrm{~dB}$ & $42 \mathrm{Mbps}$ & $168 \mathrm{Mbps}$ \\
128-QAM & $7 \mathrm{bps} / \mathrm{Hz}$ & $31.10 \mathrm{~dB}$ & $49 \mathrm{Mbps}$ & $196 \mathrm{Mbps}$ \\
256-QAM & $8 \mathrm{bps} / \mathrm{Hz}$ & $33.78 \mathrm{~dB}$ & $56 \mathrm{Mbps}$ & $224 \mathrm{Mbps}$ \\
\hline
\end{tabular}

Moreover, the transmitted signal suffers deep fades and hence microwave links are susceptible to outage events. To overcome this issue, modern microwave systems employ adaptive modulation and coding, which both have been proven to considerably enhance link performance [14]. To keep the BER performance, this technique entails the variability of the link capacity. One of the first study of network optimization assuming the above paradigm was presented in [12].

In the following, we exploit the capability of the above considered networks to adapt the capacity of links to meet the current SNR requirement. We assume that a list of states, typically corresponding to degraded weather condition in a particular area, is given. Each state is represented by a vector of link capacities where a set of links (corresponding to the degraded area) have their capacity decreased due to the modulation scheme applied to cope with the weather conditions in the area, while the others remain unchanged. Such an approach leads us to present an extension of EFR that takes into account partial link failures. The extension is discussed in detail in the following section.

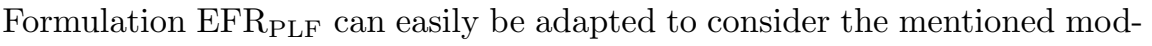
ulation schemes. In addition to notations used for EFR $\mathrm{PLF}_{\mathrm{P}}$, we introduce new 
binary optimization variables $y_{e}^{w}$ taking value 1 iff frequency $w \in W_{e}$ is selected for link $e$, where $W_{e}$ is a set of frequencies available on link $e$. The capacity of link $e$, denoted by $y_{e}$, is determined by the combination of the mod-

ulation and the frequency. It is expressed as $y_{e}=\sum_{w \in \mathcal{W}_{e}} b_{e}^{w 0} y_{e}^{w}$, where $b_{e}^{w 0}$ is the bandwidth efficiency coefficient used in optimal conditions, while in degraded conditions (partial failure state $s$ ) this is given by $b_{e}^{w s}<b_{e}^{w 0}$, which leads to decreased link capacities. Hence, the objective function of $\mathrm{EFR}_{\mathrm{PLF}^{-}}$ $\mathrm{P}$ becomes $\min C=\sum_{e \in \mathcal{E}} \sum_{w \in \mathcal{W}_{e}} \xi_{e}^{w} y_{e}^{w}$. As capacities now depend on the modulation, the right hand side of constraints $(12 \mathrm{~b})$ and $(12 \mathrm{~d})$ are to be replaced by $\sum_{w \in \mathcal{W}_{e}} b_{e}^{w 0} y_{e}^{w}$ and $\sum_{w \in \mathcal{W}_{e}} b_{e}^{w s} y_{e}^{w}$ respectively. Finally, constraints $\sum_{w \in \mathcal{W}_{e}} y_{e}^{w}=1, \quad e \in \mathcal{E}$ ensure that a link can only use one frequency.

\section{Numerical results}

In this section, we present results of a computational study illustrating the performance of EFR-P and the heuristic on the total single link failure case followed by preliminary results for the $\mathrm{EFR}_{\mathrm{PLF}}$ problem.

\subsection{Dimensioning cost}

We consider undirected networks; thus, the optimization problems are treated in their versions for undirected network graphs. Network instances used in our tests, listed in Table 2, are taken from SNDlib [10]. In the table depicted are the number of vertices, edges and demands of each networks, as well as the maximum and minimum degree of the corresponding graphs $(\Delta(G), \delta(G))$. When solving the problems, we consider sets of all paths for polska, nobel-us, and nobel-germany (as these are low meshed network instances), and all paths with up to 7 links for di-yuan (a highly meshed network with 11 nodes). The study was carried out on a computer equipped with an Intel Xeon CPU E5-2670 2.60GHz processor and $132 \mathrm{~GB}$ of RAM, using CPLEX 12.5 (CPLEX 2013).

We recall that although in theory EFR-P gives the exact formulation and solving it should provide the optimal solution of the problem, using big- $M$ coefficients in the formulation yields numerical issues. To handle this, constraints with the big- $M$ coefficients are replaced by appropriate CPLEX indicator constraints.

Table 2: Network description.

\begin{tabular}{lccccc}
\hline network & $|\mathcal{V}|$ & $|\mathcal{E}|$ & $|\mathcal{D}|$ & $\Delta(G)$ & $\delta(G)$ \\
\hline \hline di-yuan & 11 & 42 & 22 & 9 & 7 \\
polska & 12 & 18 & 66 & 5 & 2 \\
nobel-us & 14 & 21 & 91 & 6 & 2 \\
nobel-germany & 17 & 26 & 121 & 4 & 2 \\
\hline
\end{tabular}


Below we compare the cost effectiveness of the following traffic protection strategies: Elastic Flow Rerouting (EFR), Path Diversity (PD), Restricted Restoration (RR), i.e., end-to-end flow rerouting with stub release, and Global Rerouting (GR). Recall that in a failure state, GR is allowed to restore flows for all demands in the available link capacity from scratch, while $R R$ can restore only affected flows, i.e., the nominal flows that use a particular failing link in a given state.

In the reported experiments, we always use the same traffic reduction ratio $\beta$ for all demands in all failure states, i.e., $h_{d}^{s}=\beta h_{d}^{0}$ for all $d \in \mathcal{D}$ and $s \in \mathcal{S}$. We also assume that the decreasing factor $a$ is always equal to 1.0, while the increasing factor $b$ takes values $0.1,0.5,1.0$, or 3.0, which means that the nominal flows can be increased by up to $10 \%$ (resp. to $50 \%$, multiplied by 2 , or multiplied by 4). The decreasing and increasing factors are maximum limits applied to each path-flows in case of failures. In our experiments, we have incorporated all potential paths in the initial path set.

Let $C_{G R}$ denote the optimal value of the objective function (i.e., minimum cost of the link capacity) for $\mathrm{GR}$, and $C_{E F R}^{b}, C_{P D}$, and $C_{R R}$ the respective values for the remaining strategies. Note that $C_{E F R}^{a}$ is computed by solving EFR-P for the assumed thickening factor $b$. The rest of the costs are computed through solving appropriate optimization problems that can be found for example in [1]. Certainly, the computed link capacities (and thus the link capacity cost) ensure routing of all traffic demands in the nominal state and the guaranteed traffic restoration (specified by $\beta$ ) in all failure states. As we already mentioned, the cost $C_{G R}$, indicated by GR, is not greater than any of the remaining costs, as GR assumes the least restricted flow restoration mechanism. Hence, we can define the relative cost increase for each strategy $\mathrm{S}$ (where $\mathrm{S}$ stands for EFR, $\mathrm{PD}, \mathrm{RR}$, or GR) with respect to $\mathrm{GR}$ as $G_{S}=\frac{C_{S}-C_{G R}}{C_{G R}} \times 100 \%$. In the following, this quantity will be called the relative gap. Note that, by definition, $G_{G R}=0$.

In Table 3, for all four network instances, we present $G_{R R}, G_{P D}$, and $G_{E F R}^{a}$, as a function of the traffic reduction ratio $\beta$ assumed for the failure states. We consider 5 scenarios with different values of $\beta$ varying from $60 \%$ to $100 \%$. In the table, numbers in bold correspond to the scenarios, in which EFR performs better than RR and PD in terms of the cost. Experiments for values of $\beta$ less than $60 \%$ show that for EFR and GR the cost does not increase comparing to the cost of routing the demands in the nominal state.

In Table 3, interesting results in terms of the cost-effectiveness are indicated. As expected, the cost of the network for EFR is often lower than $C_{R R}$ for $b=1$ for most of $\beta$. In fact, $C_{R R}$ can be seen as $C_{E F R}^{+\infty}$ with $a=0$. Hence, because increasing $b$ decreases $C_{E F R}^{b}$, the gain of cost using EFR instead of RR comes from the ability to decrease the bandwidth of paths of unaffected demands. We also notice that the gap between the lower bound-(the cost of $C_{G R}$ ) and $C_{E F R}^{3.0}$ remains low, with a maximum of $12.6 \%$.

We now study the efficiency of the heuristic method expressed as a relative gap between the objective function of the EFR-P problem using formulation (1) (denoted $C_{E F R}^{b}$ ) and the cost achieved by the heuristic algorithm $C_{E F R-H}^{b}$. 
Table 3: Relative gap (in \%) for all strategies.

\begin{tabular}{|c|c|c|c|c|c|c|c|}
\hline & $\beta$ & $G_{R R}$ & $G_{P D}$ & $G_{E F R}^{0.1}$ & $G_{E F R}^{0.5}$ & $G_{E F R}^{1.0}$ & $G_{E F R}^{3.0}$ \\
\hline \multirow{5}{*}{ 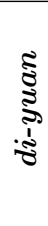 } & 0.6 & 16.5 & 41.0 & 37.2 & 27.3 & 20.5 & 10.2 \\
\hline & 0.7 & 19.4 & 47.8 & 43.4 & 31.9 & 23.9 & 11.9 \\
\hline & 0.8 & 21.0 & 53.1 & 48.0 & 34.9 & 25.9 & 12.6 \\
\hline & 0.9 & 17.5 & 60.1 & 46.9 & 31.6 & 22.0 & 11.4 \\
\hline & 1.0 & 9.4 & 60.2 & 46.0 & 30.9 & 19.7 & 11.0 \\
\hline \multirow{5}{*}{$\frac{0}{\frac{0}{20}}$} & 0.6 & 14.4 & 47.2 & 35.5 & 20.6 & 15.5 & 7.7 \\
\hline & 0.7 & 17.6 & 66.3 & 52.1 & 21.8 & 15.9 & 7.6 \\
\hline & 0.8 & 10.5 & 69.0 & 53.6 & 17.3 & 8.1 & 1.8 \\
\hline & 0.9 & 4.1 & 69.0 & 53.6 & 15.5 & 4.9 & 1.3 \\
\hline & 1.0 & 0.2 & 69.0 & 53.6 & 14.5 & 4.1 & 1.2 \\
\hline \multirow{5}{*}{ 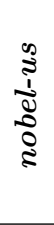 } & 0.6 & 14.8 & 59.6 & 48.1 & 31.0 & 23.3 & 11.9 \\
\hline & 0.7 & 15.9 & 77.9 & 62.8 & 33.3 & 24.2 & 11.4 \\
\hline & 0.8 & 12.5 & 88.3 & 70.6 & 31.9 & 19.7 & 7.4 \\
\hline & 0.9 & 5.8 & 88.3 & 71.7 & 34.1 & 20.5 & 11.2 \\
\hline & 1.0 & 1.9 & 88.3 & 71.7 & 33.6 & 16.9 & 8.1 \\
\hline \multirow{5}{*}{ 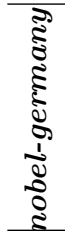 } & 0.6 & 14.1 & 44.0 & 31.4 & 17.0 & 12.1 & 6.0 \\
\hline & 0.7 & 10.7 & 51.8 & 38.3 & 19.1 & 15.2 & 12.5 \\
\hline & 0.8 & 5.0 & 51.9 & 39.7 & 15.5 & 10.2 & 6.6 \\
\hline & 0.9 & 1.6 & 51.9 & 39.7 & 14.8 & 8.0 & 4.1 \\
\hline & 1.0 & 0.0 & 51.9 & 39.7 & 14.6 & 5.9 & 3.0 \\
\hline
\end{tabular}

Table 4 shows the values of $H_{E F R}^{b}=\frac{C_{E F R-H}^{b}-C_{E F R}^{b}}{C_{E F R}^{b}} \times 100 \%$ - the relative gap between $C_{E F R-H}^{b}$ and $C_{E F R}^{b}$-as a function of the traffic reduction ratio. The results are given for the settings used in Table 3. We notice that the heuristic method performs well for all networks indicating the maximum relative gap of less than $2 \%$. However, as this method contains some randomness, the gap with the exact solution can be different if we compute the solution of the heuristic a second time. Still, the heuristic seems to be able to find a solution close to the optimal solution, with a gap not exceeding $2 \%$ in most cases. In the table, we present the best obtained gap after running the heuristic for three times. Thus, we notice that the heuristic is usually able to find the optimal solution after just a few repetitions, as indicated by the 0.0 value of the gap (in bold).

Finally, we study the performance of $\mathrm{EFR}_{\mathrm{PLF}}$, i.e., EFR applied to partial failures. Let $C_{G R}$ denote the optimal value of the objective function (i.e., minimum cost of the link capacity) for GR, and $C_{E F R_{\mathrm{PLF}}}^{b}, C_{F T}$ the respective values for the remaining strategies. We define the relative cost increase for each strategy $\mathrm{S}$ (where $\mathrm{S}$ stands for $E F R_{\mathrm{PLF}}$ or FT) with respect to GR as $K_{S}=\frac{C_{S}-C_{G R}}{C_{G R}} \times 100 \%$. To make a comparison with results for FT and GR 
Table 4: Relative gap (in \%) between the exact solution and the heuristic solution.

\begin{tabular}{|c|c|c|c|c|c|}
\hline & $\beta$ & $H_{E F R}^{0.1}$ & $H_{E F R}^{0.5}$ & $H_{E F R}^{1.0}$ & $H_{E F R}^{3.0}$ \\
\hline \multirow{5}{*}{ 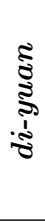 } & 0.6 & 0.0 & 0.0 & 0.0 & 0.0 \\
\hline & 0.7 & 0.0 & 0.0 & 0.0 & 0.0 \\
\hline & 0.8 & 0.0 & 0.0 & 0.0 & 0.0 \\
\hline & 0.9 & 0.0 & 0.0 & 0.0 & 0.0 \\
\hline & 1.0 & 0.0 & 0.0 & 0.0 & 0.0 \\
\hline \multirow{5}{*}{ 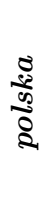 } & 0.6 & 0.0 & 0.0 & 0.0 & 0.0 \\
\hline & 0.7 & 0.0 & 0.9 & 0.4 & 0.2 \\
\hline & 0.8 & 0.0 & 0.6 & 0.7 & 0.0 \\
\hline & 0.9 & 0.0 & 1.1 & 0.7 & 0.0 \\
\hline & 1.0 & 0.0 & 1.2 & 0.1 & 0.0 \\
\hline \multirow{5}{*}{ 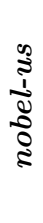 } & 0.6 & 0.0 & 0.0 & 0.0 & 0.0 \\
\hline & 0.7 & 0.0 & 0.0 & 0.0 & 0.0 \\
\hline & 0.8 & 0.0 & 0.0 & 0.0 & 0.1 \\
\hline & 0.9 & 0.0 & 0.0 & 0.1 & 0.1 \\
\hline & 1.0 & 0.0 & 0.1 & 0.1 & 0.1 \\
\hline \multirow{5}{*}{ 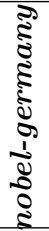 } & 0.6 & 0.0 & 0.0 & 0.0 & 0.0 \\
\hline & 0.7 & 1.1 & 0.0 & 0.0 & 0.0 \\
\hline & 0.8 & 0.0 & 0.0 & 0.0 & 0.0 \\
\hline & 0.9 & 0.0 & 0.0 & 0.0 & 0.0 \\
\hline & 1.0 & 0.0 & 0.1 & 0.3 & 0.0 \\
\hline
\end{tabular}

possible, some vital changes are needed in the way the capacities are affected by the perturbation for $\mathrm{EFR}_{\mathrm{PLF}}$. Instead of variables $y_{e}^{w}, e \in \mathcal{E}, w \in \mathcal{W}_{e}$ we use $y_{e}, e \in \mathcal{E}$. By extension, instead of $b_{e}^{w s} y_{e}^{w}, e \in \mathcal{E}, w \in \mathcal{W}_{e}, s \in \mathcal{S}$ we use $\gamma y_{e}, e \in \mathcal{E}, s \in \mathcal{S}$, where $\gamma$ express the perturbation ratio. The results are given for several levels of perturbation $\gamma: 50 \%, 75 \%$, and $95 \%$. As no network instance could be solved within a limited period of time (10 hours), we present results of network polska with a limited set of demands: 20 demands instead of 66 .

We notice that EFR PLF performs generally better than FT. For lower perturbation ratios, for instance $\gamma=50 \%$, differences of costs of the network for FT and EFR PLF $_{\text {remains insignificant - the gap between FT and EFR }}$ PLF for all values of $b$ is less than $10 \%$. However, if we consider stronger perturbation

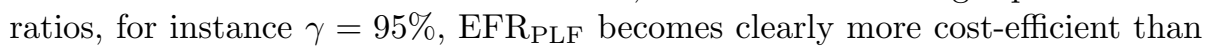
FT. When the thickening ratio is very low, for instance $10 \%$, the gap between FT and EFR $\mathrm{ELF}_{\mathrm{F}}$ is equal to $5.7 \%{ }^{2}$. If we increase the thickening ratio, the gap between FT and EFR $\mathrm{PLF}_{\text {reaches }} 25.7 \%(b=3)$.

\footnotetext{
${ }^{2}$ This gap value is computed using results for each strategy as done for the gap with GR.
} 


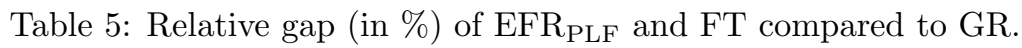

\begin{tabular}{crrrrr}
\hline$\gamma$ & $K_{F T}$ & $K_{E F R}^{0.1}$ & $K_{E F R}^{0.5}$ & $K_{E F R}^{1.0}$ & $K_{E F R}^{3.0}$ \\
\hline \hline $50 \%$ & 14.3 & 11.3 & 6.6 & 5.0 & 3.3 \\
$75 \%$ & 26.0 & 20.9 & 10.9 & 7.8 & 3.9 \\
$95 \%$ & 43.9 & 35.8 & 18.3 & 11.6 & 7.0 \\
\hline
\end{tabular}

\subsection{Restoration time}

We analyze here the solutions obtained with the EFR, GR, and RR strategies in terms of average number of paths, length of the paths, and restoration time. Table 6 shows the average number of paths in the nominal state, the total number of distinct paths, and the number of paths used for thickening or new paths created. Notice that not all strategies allow for establishing new paths. Moreover, in the table, the average length of the paths is shown both for nominal and failure states. We notice that even though GR and RR use less paths in the nominal state, the average total number of paths used by these strategies is significantly higher.

Table 6: Paths of the solutions obtained by EFR, GR, and RR for network

$$
\text { polska }
$$

\begin{tabular}{lcccc}
\hline & $E F R^{0.5}$ & $E F R^{1.0}$ & $G R$ & $R R$ \\
\hline \hline Avg. number of paths per demand in nominal state & 4.12 & 3.02 & 1.27 & 1.51 \\
Avg. number of distinct paths per demand & 4.12 & 3.02 & 7.26 & 7.36 \\
Avg. number of paths to thick/create per demand & 0.94 & 0.61 & 1.24 & 0.41 \\
Avg. length of the paths in nominal state & 3.41 & 3.14 & 3.13 & 2.46 \\
Avg. length of all paths & 3.41 & 3.14 & 4.44 & 5.57 \\
\hline
\end{tabular}

In the following, we study the restoration time of EFR, compared to GR and RR. In the experiment, we use a simplified restoration model that returns a fraction of satisfied demands in a function of time elapsed after the failure. The results are presented in Figures 5 and 6, where the former represents the ratio of satisfied demands in time in the network polska for a total failure of a link at the border of the network, while the latter presents the same result for a total failure of a link in the center of the network.

As expected, EFR leads to a substantial gain in terms of restoration time, roughly two times faster than GR and three times faster than RR. This is mainly due to two factors: EFR do not create new paths and the total number of paths it uses is smaller. Therefore, the time when EFR practically ends the whole restoration process is also the time when GR strategy ends disconnecting 


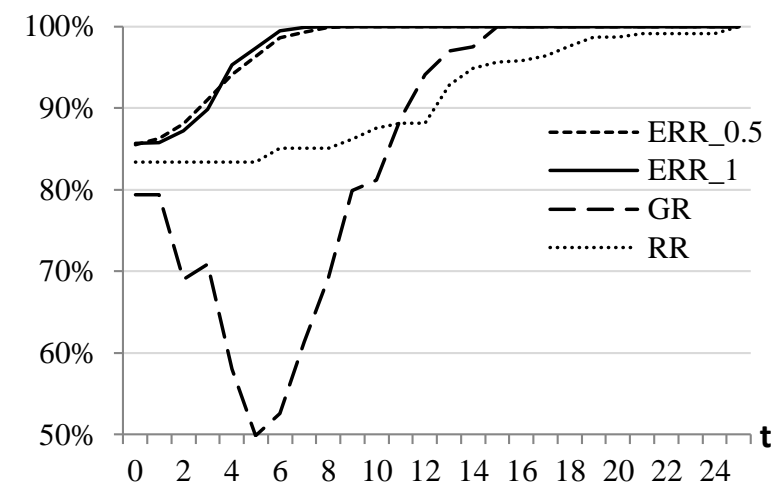

Figure 5: Demand satisfaction ratio of the network polska after failure of Kolobrzeg-Szczecin (at the border of the network).

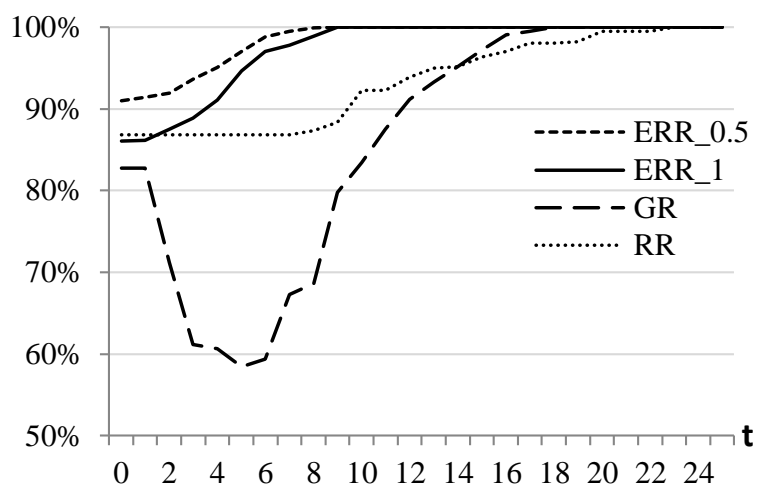

Figure 6: Demand satisfaction ratio of the network polska after failure of Lodz-Warsaw (in the center of the network).

flows that are to be rerouted. The reason GR outperforms RR in terms of total restoration time is the average length of restoration paths used by the strategies. The longer restoration paths of RR strategy induce longer restoration times. Another important aspect, mentioned earlier, is the capability of EFR to maintain a high level of satisfaction ratio of demands during the restoration process. From the figures we notice that GR encumbers the process as a lot of routing paths should first be released and others have to be settled from the scratch, while EFR benefits from optimized flow paths at a large part available in most of failure situations. The issue can be perfectly seen in the figures around time 5-7, when the demand satisfaction ratio for GR plunges to merely $50 \%$, while at the same time reaching $100 \%$ for EFR and about $85 \%$ for RR. This feature is expected to be even more important for partial failure situations. 


\section{Concluding remarks}

In the paper, we have studied a protection strategy called Elastic Flow Rerouting

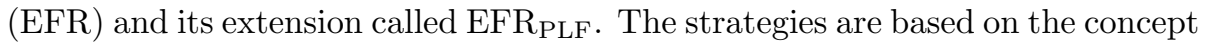
of elastic (failure state-dependent) path-flows. EFR is designed to handle total link failures, while its extension deals with multiple partial link failures related to broadband wireless (FSO, microwave) networks. When it comes to the relation of link capacity cost, management effort, and traffic restoration time, the EFR strategy appears to be encouraging, especially when traffic is supposed to be partially restored.

From the theoretical viewpoint, optimization of EFR gives rise to a challenging multi-commodity flow problem EFR-P, combining two difficult issues: a combinatorial subproblem dealing with the choice of the demand states, and a likely $\mathcal{N} \mathcal{P}$-hard pricing subproblem for path generation. To the best of our knowledge, such a problem has not been considered in the literature on traffic protection strategies before. We have introduced an exact MIP formulation of EFR-P that works for a given set of admissible paths, and proposed a fast suboptimal heuristic approach for EFR-P. Looking for an exact approach with a reasonable efficiency will be the subject of our future work.

We have also considered the case with partial failures. The corresponding problem, denoted by EFR $\mathrm{PLF}_{\mathrm{P}} \mathrm{P}$, is even more difficult, since it combines two levels of decisions: at the first level it choses flows that have to be thinned and at the second level it optimizes the way the affected demands can enlarge their flows using resources released from the unaffected demands. All this makes the problem highly combinatorial. Additionally, the corresponding pricing problem is very complicated, as it embraces features coming from two other $\mathcal{N} \mathcal{P}$-hard pricing problems: one for FT and the other for EFR.

\section{Acknowledgement}

The work of the French authors was carried out in the framework of the Labex MS2T, which was funded by the French Government, through the program " Investments for the future managed by the National Agency for Research (Reference ANR-11-IDEX-0004-02). M. Pióro and M. Żotkiewicz were supported by the FP7 project IDEALIST (grant agreement no. 317999), and by National Science Centre, Poland (grant 2011/01/B/ST7/02967). Also, the authors wish to thank Dmitri Papadimitriou for his enlightening comments.

\section{Appendix: Binary formulation of the pricing prob- lem}

The pricing problem formulated in (11) is difficult because of the particular form of the total dual length $\|q\|$. Nevertheless, the problem can be stated as a binary programming problem by means of formulation (14) given below. In the 
formulation, binary variables $u_{e}, e \in \mathcal{E}$, specify the path $q$ we are looking for: $q=\left\{e \in \mathcal{E}: u_{e}=1\right\}$. Binary variables $z^{s}, s \in \mathcal{S}$, in turn, denote if $|p|^{s}>\lambda_{d}^{s}$. If that is the case, then $z^{s}=1$; otherwise $z^{s}=0$. Besides, $\delta^{+}(v)$ and $\delta^{-}(v)$ denote the sets of all links outgoing from, and all links incoming to, respectively, node $v \in \mathcal{V}$, constant $d$ indicates a considered demand, node $o$ is its originating node and $t$ is its terminating node.

$$
\begin{array}{r}
\min L=\sum_{e \in \mathcal{E}} u_{e} \pi_{e}^{0}+\sum_{s \in \mathcal{S}}\left(1-u_{s}\right)\left(\sum_{e \in \mathcal{E}} u_{e} \pi_{e}^{s}-\lambda_{d}^{s}\right)+ \\
-a \sum_{s \in \mathcal{S}} z^{s}\left(1-u_{s}\right)\left(1-T_{d}^{s}\right)\left(\sum_{e \in \mathcal{E}} u_{e} \pi_{e}^{s}-\lambda_{d}^{s}\right)+ \\
-b \sum_{s \in \mathcal{S}}\left(1-z^{s}\right)\left(1-u_{s}\right) T_{d}^{s}\left(\sum_{e \in \overline{\mathcal{E}}} u_{e} \pi_{e}^{s}-\lambda_{d}^{s}\right) \\
\sum_{e \in \delta^{+}(o)} u_{e}-\sum_{e \in \delta^{-}(o)} u_{e}=1 \\
\sum_{e \in \delta^{+}(v)} u_{e}-\sum_{e \in \delta^{-}(v)} u_{e}=0, \quad v \in \mathcal{V} \backslash\{o, t\} \\
\lambda^{s}\left(1-z^{s}\right) \geq \lambda^{s}-\sum_{e \in \mathcal{E}} \pi_{e}^{s} u_{e}, \quad s \in \mathcal{S} \\
z^{s} \sum_{e \in \mathcal{E}} \pi_{e}^{s} \geq \sum_{e \in \mathcal{E}} \pi_{e}^{s} u_{e}-\lambda^{s}, \quad s \in \mathcal{S} \\
u_{e} \in\{0,1\}, e \in \mathcal{E} ; z^{s} \in\{0,1\}, \quad s \in \mathcal{S} .
\end{array}
$$

Constraints (14b) and (14c) assure that variables $u_{e}$ that are equal to 1 form a path from $o$ to $t$. Constraints $(14 \mathrm{~d})$ and $(14 \mathrm{e})$ force each variable $z^{s}, s \in \mathcal{S}$, to be equal to 1 when the length, with respect to $\pi^{s}$, of the path $q$ defined by variables $u$ is greater than $\lambda^{s}$; and to be equal 0 otherwise. $T_{d}^{s}$ are already given from the instance of the problem in hand.

To get rid of bi- and tri-linearities involving variables $z^{s}$ and $u_{e}$ in the objective function, we can introduce five binary (formally continuous) variables $W^{s}, V^{s}, X_{e}^{s}, Y_{e}^{s}, Z_{e}^{s}, e \in \mathcal{E}, s \in \mathcal{S}$. The objective function should be rewritten as

$$
\begin{array}{r}
L=\sum_{e \in \mathcal{E}} u_{e} \pi_{e}^{0}+\sum_{s \in \mathcal{S}}\left(\sum_{e \in \mathcal{E}} X_{e}^{s} \pi_{e}^{s}-\left(1-u_{s}\right) \lambda_{d}^{s}\right)+ \\
-a \sum_{s \in \mathcal{S}}\left(1-T_{d}^{s}\right)\left(\sum_{e \in \mathcal{E}} Y_{e}^{s} \pi_{e}^{s}-W_{e}^{s} \lambda_{d}^{s}\right)-b \sum_{s \in \mathcal{S}} T_{d}^{s}\left(\sum_{e \in \overline{\mathcal{E}}} Z_{e}^{s} \pi_{e}^{s}-V_{e}^{s} \lambda_{d}^{s}\right)
\end{array}
$$

and add the following set of constraints

$$
\begin{array}{r}
X_{e}^{s} \geq u_{e}-u_{s}, \quad e \in \mathcal{E}, \quad s \in \mathcal{S} \\
Y_{e}^{s} \leq z^{s}, Y_{e}^{s} \leq\left(1-u_{s}\right), Y_{e}^{s} \leq u_{e}, \quad e \in \mathcal{E}, \quad s \in \mathcal{S} \\
Z_{e}^{s} \leq\left(1-z^{s}\right), Z_{e}^{s} \leq\left(1-u_{s}\right), Z_{e}^{s} \leq u_{e}, \quad e \in \mathcal{E}, \quad s \in \mathcal{S} \\
W^{s} \geq z^{s}-u_{s}, \quad s \in \mathcal{S} \\
V^{s} \geq 1-u_{s}-z^{s}, \quad s \in \mathcal{S} .
\end{array}
$$




\section{References}

[1] M. Pióro and D. Medhi, Routing, Flow, and Capacity Design in Communication and Computer Networks, Morgan Kaufman, San Francisco, 2004.

[2] S. Orlowski and M. Pióro, Complexity of column generation in network design with path-based survivability mechanism, Networks, 59(1):132-147, 2012.

[3] A. M. C. A. Koster, A. Zymolka, M. Jager, R. Hulsemann, and C. Gerlach, Demand-wise shared protection for meshed optical networks, Journal of Network and Systems Management, 13(1):35-55, 2005.

[4] I. Shinko, Y. Fouquet, and D. Nace, A study on a distributed rerouting scheme, In Proc. of NetMM workshop, AINA, 2013.

[5] Y. Fouquet, D. Nace, M. Pióro, and I. Shinko, Elastic Routing: a distributed variant, implementation issues, and numerical results, In Proc. of 3PGCIC, 2013.

[6] J.-F. Maurras and S. Vanier, Network synthesis under survivability constraints, 4OR, 2(1):53-67, 2004.

[7] A. Mereu, D. Cherubini, A. Fanni, and A. Frangioni, Primary and backup paths optimal design for traffic engineering in hybrid IGP/MPLS networks, In Proc. of DRCN, 2009.

[8] S. Orlowski, Local and global restoration of node and link failures in telecommunication networks, Diploma thesis, Konrad-Zuse-Zentrum fr Informationstechnik Berlin (ZIB), 2003, Berlin, Germany.

[9] D. Nace, M. Pióro, A. Tomaszewski, and M. Żotkiewicz, Complexity of a classical flow restoration problem, Networks, 62(2):149-160, 2013.

[10] S. Orlowski, R. Wessäly, M. Pióro, and A. Tomaszewski, SNDlib 1.0 Survivable network design library, Networks, 55(3):276-286, 2010.

[11] H. Anderson. Fixed Broadband Wireless System Design, J.Wiley \& Sons, 2003.

[12] G. Classen, D. Coudert, A. Koster, and N. Nepomuceno. A chanceconstrained model and cutting planes for fixed broadband wireless networks. In Proc. INOC, 2011.

[13] Y. Fouquet, D. Nace, M. Pióro, M. Poss and M. Żotkiewicz. Elastic Rerouting - models and resolution methods. In Proc. RNDM, 2014.

[14] A. Goldsmith and S. Chua. Variable-rate variable-power MQAM for fading channels. IEEE Trans. Commun, 45(10):1218-1230, 1997. 
[15] H. Lehpamer. Microwave Transmission Networks: Planning, Design, and Deployment, McGraw-Hill, 2010.

[16] M. Pióro, D. Nace, and Y. Fouquet. An optimization model for communication networks with partial multiple link failures. In Proc. RNDM, 2013.

[17] I. K. Son and S. Mao. Design and optimization of a tiered wireless access network, In Proc. IEEE INFOCOM, pp. 1-9, 2010.

[18] A. Tomaszewski, M. Pióro, and M. Żotkiewicz. On the complexity of resilient network design. Networks, 55:109-118, 2010.

[19] H. Willebrand and B. S. Ghuman. Free Space Optics: Enabling Optical Connectivity in Today's Networks, Sams Publishing, 2002.

[20] A. Tomaszewski. The final answer to the complexity of a basic problem in resilient network design, In Electronic Notes in Discrete Mathematics, 41:455-462, 2013.

[21] S. Fortune, J. Hopcroft, J. Wyllie. The directed subgraph homeomorphism problem. Technical Report, Ithaca, NY, USA, 1978. 